\title{
Mismatch losses minimization in photovoltaic arrays by arranging modules applying a genetic algorithm
}

\begin{abstract}
Photovoltaic (PV) arrays consist of series and parallel connections of PV modules. Difference in current-voltage (I-V) characteristics among a batch of modules form an array causes power losses in PV systems referred to as mismatch losses. These power losses are conventionally reduced by module sorting techniques which sort modules based on an I-V parameter such as short circuit current, current at maximum power or maximum power. This work introduces a new method that employs genetic algorithm (GA) to find an arrangement of modules in an array which minimizes mismatch losses more effectively than conventional methods do. Extensive simulations are applied to adapt a GA to the problem of mismatch losses, find the arrangement and demonstrate its superiority over module sorting techniques in terms of mismatch losses decrement and energy yield increment. Instructions for practical application of the suggested method are also provided.
\end{abstract}

Keyword: Mismatch losses; Genetic algorithm; Module sorting techniques; Photovoltaic 